\title{
Ripples of Change - An AI Job Crafting Model for Human-in-Control
}

\author{
Rania Afiouni \\ McGill University \\ rania.afiouni@mail.mcgill.ca
}

\author{
Alain Pinsonneault \\ McGill University \\ alain.pinsonneault@mcgill.ca
}

\begin{abstract}
Introducing a new Artificial Intelligence (AI) system disrupts workers' sense of control. To restore it, individual workers are likely to engage in selfinitiated changes to their jobs. We build on job crafting theory and extend it to propose a theoretical model explaining the ripple effect of changes from tasks to skills, relationships, and finally job cognition. We introduce the concept of human-in-control (one's perception of their ability to deliver desired work outcomes in a work context involving AI) as the goal of the job crafting process. Our work provides a novel and important perspective on adaptation to AI through job transformation. As such, it opens numerous avenues for research in this nascent stream.
\end{abstract}

\section{Introduction}

The introduction of a new Information System (IS) in a work environment is often seen in IS research as a disruption that calls for some form of adaptation. Salient examples are the research streams of acceptance $[1,2]$, resistance $[3,4]$, adaptation $[5,6]$, coping $[7,8]$, and many studies in IS use in general. In this literature, IS disruption is found to influence practices [9], power balance [4], habits [10], and routines [11] to name a few. We argue that these seemingly distinct though interrelated responses to disruption share a common denominator: an effort exerted by individuals to keep or regain a sense of control over their environment.

Feeling a loss of control can manifest in different ways, many of which are indirectly covered in extant adaptation to IT literature. For instance, holding on to old ways of doing work [12] or retrofitting the new system to mimic the old one [13] can be viewed as attempts to maintain the familiar tools that help keep a control perception. The same can be said of reducing the use of the new system or even avoiding it [14]. Similarly, appropriating a system to improve the use experience [15] and innovating with the new technology [16] allow users to restore and possibly gain a sense of control.

Restoring the sense of control cannot happen without change. This change is typically studied in IS literature as either adapting the technology to the user's desires or adapting the self to the technology. However, this perspective on adaptation does not fully explain the change associated with an AI context. With regard to the former, unlike traditional IS, AI with its dynamic rules and heuristic nature allows little if any room for deterministic change to the artifact through customization and personalization. As for the latter adaptation, the agentic nature of the AI artifact and the increased complexity of the tasks it replaces suggest a radical change in human work altering not only tasks but also work design. Therefore, the passive perspective of humans adapting to the technology is not enough anymore. Instead, a more relevant one is to study how work designs can be adapted to better meet human needs in a context of task sharing between human and AI [17].

Adapting work design to restore the sense of control is likely to be especially important in the context of Artificial Intelligence (AI) systems due to the technology's fundamental characteristics. The heuristic nature of the technology where the algorithmic processing between input and output evolves with training and use [18] gives AI the ability to address complex tasks that are high on uncertainty and cognition [19]. Equipped with these capabilities, the machine is therefore an actor able to exhibit a nonhuman behavior of its own, which is dynamic and evolutionary [20]. Rich of this machine behavior, AI artifacts are agentic ones that produce agentic ambiguity in a work environment [21], therefore raising multiple questions of which control is an important one.

The importance of control is further emphasized in a context of task automation where the human remains accountable. While not every task can be delegated to machines, for some, AI has reached better performance accuracy than humans. As a result, AI is being integrated into the work of higher skill workers 
with critical responsibilities and is becoming increasingly autonomous. However, these workers are still accountable for the end results of their work and are legally responsible for any harm the machine might cause [20]. An accountable worker using an agentic AI artifact is therefore likely to feel more strongly the disruption to their sense of control and the need to restore it.

One approach that we adopt to study work redesign to restore control is job crafting theory where workers actively change multiple elements of their job and themselves in ways that positively influence them at work [22]. We thereby explain in the next section what job crafting is and its relation to AI and control. We then build on job crafting in section 3 to develop a theory that explains how, motivated by their need for control, individuals redesign their job in light of an AI disruption in order to reach a perception of human-incontrol (one's perception of their ability to deliver desired work outcomes in a work context involving AI). We then conclude with a discussion of implications and opportunities for future research.

\section{Theoretical foundation: Job crafting}

In giving attention to change by workers to their work, we acknowledge that job design is not exclusively a top-down process and that employees have and exert agency in the design of their own jobs. This idea is best depicted by job crafting theory which we explain, connect with AI, and complement with a more elaborate control concept in the following three paragraphs.

\subsection{Job crafting theory}

According to job crafting theory, employees are proactive agents of their work who can redesign their jobs in a bottom-up approach [22, 23], thereby supplementing management driven job design [24]. Job crafting is defined as "the physical and cognitive changes individuals make in the task or relational boundaries of their work" [22:179].

The job crafting literature proposes numerous models $[22,23,25,26]$ that are marginally different from one another, but that all present some or all of four crafting forms. The first, task crafting, involves changing the physical and temporal boundaries of the tasks performed on the job. Examples are adding, removing, or modifying tasks or giving more time and attention to some tasks than others. The second, skill crafting, is about developing new complementary skills to achieve sustainable and successful job crafting outcomes [27]. The third, relational crafting, consists in strengthening or weakening some relationships at work, whether in terms of amount or quality of interactions. Finally, the fourth is cognitive crafting and is about altering one's view of their work and their role through it.

\subsection{Job crafting fit with AI}

Technology does not affect single characteristics of a job, rather job design itself including but not limited to skills, relationships, and job demands [17]. Job crafting theory allows this wholistic view of job transformation. It is suitable for studying job change in the context of AI for several other reasons too. First, $\mathrm{AI}$ is associated with high levels of uncertainty. In particular, deep learning, a cutting-edge machine learning scheme and an increasingly dominant form of AI, exhibits black box characteristics [28]. In fact, deep learning algorithms' data driven inductive rule emergence is opaque by design. Opacity here is in the sense of an input resulting in an output with little if any understanding of the logic through which the processing happened [29]. Against such background of uncertainty and complexity, scholars have highlighted the importance of employees proactively changing their tasks and roles rather than solely relying on organizations and their management for initiating the change [30]. Second, job crafting allows adaptation to change [31], which can be a technology induced one [32] A new AI implementation is a major change and a major challenge given the particularity of the technology. Job crafting can therefore be used to study the adaptive response to this challenge through proactive behaviors [33]. Third, autonomy has been found to stimulate job crafting behavior [24]. Jobs where humans and AI share tasks typically benefit from a certain level of autonomy. Examples are auditors and financial advisors whose work requires human judgment, problem solving, and analytical skills, which all imply some autonomy. However rare, jobs that require little or none of these elements and where routine tasks make up almost their entirety are replaced rather than augmented by $\mathrm{AI}$, and job replacement is outside the scope of this study. In conclusion, we believe that job crafting theory provides a fitting lens for investigating the phenomenon at hand.

\subsection{Control in the context of AI}

In the job crafting literature, control is an important concept and a motivator for crafting behavior, but it has received little theoretical attention. Yet, the concept of control is fundamental in the context of AI. Individuals remain accountable for actions and decisions made in their job. They can be 
called on to justify an action to others, which, if the justification is unsatisfactory, will cause them negative consequences [34]. These consequences can in some cases reach legal liability, either directly or indirectly, for an AI deployment that workers have little control over and an AI decision recommendation that can be highly opaque and which premises they often do not have knowledge of [35]. In addition, notwithstanding the superior narrow intelligence of AI, humans still outperform it in complex decisions that require high level expert knowledge and instinctive judgment [36]. This is all the more true since learning in AI is well adapted for understanding the what in the data, but completely blind as to the why or the cause effect relationships leading to those patterns [37].

With the disruption of a new AI system, a gap widens between the need for control accentuated by accountability and the perceived control disrupted by delegation to AI. We therefore introduce the concept of human-in-control that we define as one's perception of their ability to deliver desired work outcomes in a work context involving AI. Human-in-control is the perception of control over the outcomes of one's work including automated and non-automated tasks as well as other elements of the job.

We turn to psychology to elaborate the concept of control and augment job crafting theory so that it better fits with the specificities of the AI context. Fundamentally, we are interested in control as being in control, a perception that is self-targeted and that can motivate individuals to craft their jobs both behaviorally and cognitively. We draw on Bryant [38] to develop the concept of control in the context of AI. The author develops a typology of control along two dimensions. The first one accounts for differences in control for perceived positive versus negative events where the former aims at reaching a positive reinforcer while the latter seeks to avoid adversity. The second dimension distinguishes between control gained by changing the environment to bring it closer to one's needs (primary control) or by bringing the self to fit more effectively with the environment (secondary control). With this distinction, much of the behavior typically seen as uncontrollability is actually a different expression of control. Reaching a sense of control is therefore not limited to blatant controlling behavior but can also be a more subtle inward and reflective process [39].

Along these two dimensions, Bryant's four-factor model of perceived control includes Obtaining

\footnotetext{
${ }^{1}$ Bryant uses the label "coping" to describe this control process. However, it is important to note that this refers to a different mechanism than the 'coping' label widely used in Coping Theory of the Psychology literature and in IS [7]. Therefore, and to avoid
}

positive outcomes (primary control and positive event), Savoring positive outcomes (secondary control and positive event), Avoiding negative outcomes (primary control and negative event), and Bearing ${ }^{1}$ negative outcomes (secondary control and negative event). In our theoretical development, we explain when and how these control processes motivate and guide job crafting in the context of a new AI introduction.

\section{Theoretical model: Ripples of job crafting}

When job crafting is applied to the context of an AI introduction, we argue that a ripple effect is triggered by the redistribution of tasks between humans and AI (figure 1). As the new technology disrupts their sense of control, individual workers attempt to restore it or gain it starting with the element that is closest to the technology, that is tasks. With new tasks being added or emphasized and others eliminated or reduced, the skill set needed to execute them changes too. Individuals then engage in skill crafting efforts building new ones and not using some existing ones. A third ripple follows as the modified tasks and skills call for altered interactions with different actors therefore weakening some relationships and strengthening others. Individuals engage here in relational crafting. Finally, a last ripple that builds on

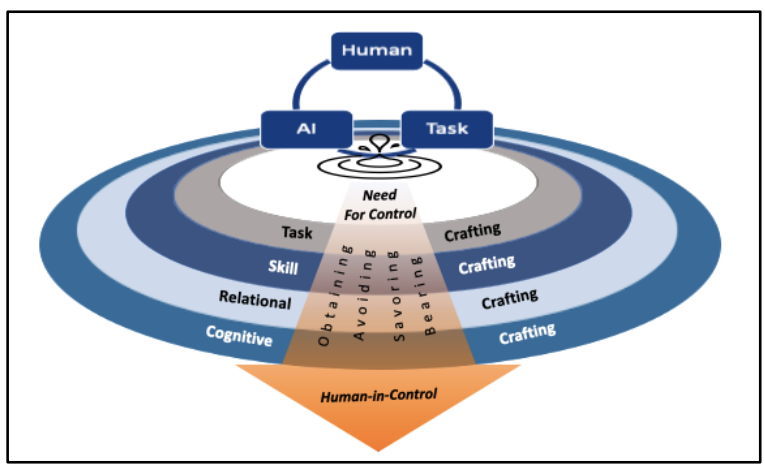

Figure 1. From need for control to human-incontrol

all three previous forms of crating is cognitive crafting where individuals develop a new understanding of what their job is.

As one evolves in the process of job crafting and moves across the ripples, the sense of control evolves too. In the next paragraphs, we go through this

confusion, we have relabeled this control process 'bearing', which equally captures its essence. 
evolution ripple by ripple and explain the rationale for the iterative nature of the job crafting process. Figure 2 illustrates these ripples with a summary of the main crafting activities identified for each.

\subsection{Ripple 1: Task crafting}

An important particularity of job crafting that sets it apart from other job design theories is that the process is a bottom-up one initiated by the worker rather than management. This implies that changes are psychology, associating trust with a positive valency and distrust with a negative one [41].

Our focus on trust is further due to its high relevance in human-AI relations given the nondeterministic AI behavior and AI's expanding role in the workplace. Cognitive factors such as bias, transparency, explainability, and reliability can contribute to this trust as well as emotional factors such as fear and anxiety [42]. However, trust is not to be understood in a restricted sense of trust in AI per se. As AI automates more diverse tasks, what is more

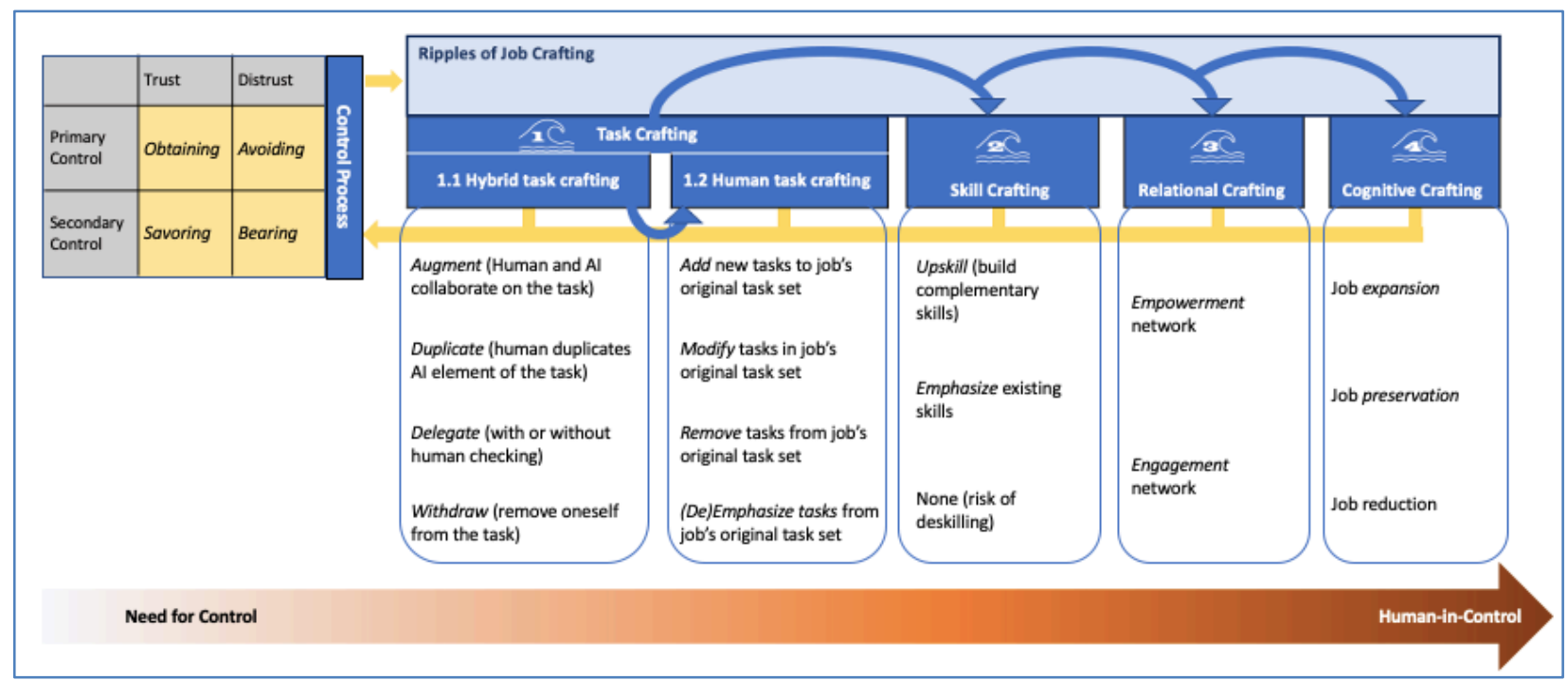

Figure 2. Ripples of job crafting for human-in-control

different from one worker to the other. In terms of tasks, what influences these differences and how do they materialize?

Building on the four-factor model of control perception, a first influence on the process followed to achieve a sense of control is whether the individual worker engages in primary or secondary control. While both coexist most of the time, primary control is salient when the self is the most powerful agent and secondary is salient when other agents are more powerful [39]. Examples of the latter are the individual's abilities or constraining contextual factors related directly or indirectly to AI.

A second influence is whether AI is perceived positively or negatively by the worker. We posit that trust and distrust in AI represent respectively positive and negative perceptions of it. Our claim is supported by a functional neuroimaging study which shows that trust activates brain areas associated with positive emotions and expectations of positive rewards, while distrust activates brain areas associated with negative emotions and expectations of negative losses [40]. This valency view of trust is also present in behavioral relevant for us is trust in AI to successfully perform a particular task, which can change from one task to another. This notion of trust includes an evaluation of reliance on AI for the task in question, which can lead or not to an action of trusting in the form of delegation [43]. This is where differences in tasks become especially important.

We differentiate first between hybrid tasks where worker and AI collaborate to reach an outcome and human tasks that do not involve AI. We further divide hybrid tasks into critical and non-critical ones since trust evaluations and consequently delegation decisions are not the same for the two given the difference in accountability.

3.1.1. Human-AI hybrid task crafting. AI perception's positive or negative valency influences decisions about hybrid tasks, how to approach them, and how much to delegate to technology. This is not only suggested in the AI literature, but also in job crafting where empirical evidence supports different job crafting behavior according to patterns of approach or promotion (both based on a desire to reach positive outcomes) on one hand, and avoidance or prevention 
(both intended to reduce threat of negative outcomes) on the other $[25,26]$. Drawing on both literatures, we have identified four potential forms of hybrid task crafting.

Augment: human and AI collaborate by combining both their intelligences in achieving complex tasks in what is referred to as hybrid intelligence [44]. When trust in AI for that task is high, human delegates to AI task elements that might be beyond human capabilities including but not limited to sensing and analyzing data [21]. Both agents coevolve, learn from each other, and complement each other's strength [45], and the human elements of tasks often increase in diversity and cognitive level $[17,46]$. Engaging in collaboration with AI through new ways of executing tasks changes the nature of work which can be associated with primary control. Augmentation is therefore likely to occur under an obtaining control process and can involve both critical and non-critical tasks. The difference is that for critical tasks, the human element is likely to comprise verifying - to the extent possible - AI outcomes. This controlling behavior has the potential to increase trust in AI when accountability is high [43].

Duplicate: Distrust leads to low reliance on AI [42], especially in a critical task context. Instead of cooperation as in augmentation, there is conflict between AI and human [21]. In situations similar to cancer diagnosis or piloting a plane, distrust results in humans ignoring the AI outcome and duplicating its task elements when possible. While not intended in the workflow design, this duplication is a form of engagement by the worker aimed at preventing AI harm [25]. It is part of a prevention oriented primary control process of avoidance. Duplication could also be applied to non-critical tasks if needed. However, low accountability might more likely lead to informal removal by the worker of either the hybrid task or its AI elements from the job's task set.

Delegate: Trust in AI and reliance on it do not always lead to augmentation. The latter is associated with collaboration on the task, but not all hybrid configurations are necessarily collaborative. Some forms of working with AI entail sequential decision making [47] where each of human and AI use the outcome of the other for their elements of the task. Such a configuration often keeps agents outside the loop of each other. Here, there is no mutual learning between AI and human. Instead, adoption of AI constitutes an additional resource for the worker [26] and a sense of control is achieved through savoring of delegation to a trustworthy AI with no ambition to change the task environment.

Withdraw: A negative distrustful perception of AI can be coupled with secondary control to bring oneself into line with the disrupted work environment. One way to achieve this is through withdrawal, which can be achieved by removing oneself mentally or physically from the hybrid task to prevent facing unfulfillable expectations [39]. The human here acknowledges AI as a more powerful agent and attempts to fit in the new work environment through distancing oneself from AI [39]. This form of passive resistance has been observed with older technologies too through delegating tasks involving use of IT to others at work [4]. While such a form of task crafting can help achieve a sense of control through a bearing control process, it carries the risk if used persistently of alienating the worker. In particular, when the tasks withdrawn from are of critical nature for one's work, this risk can possibly evolve into progressive job withdrawal [26].

3.1.2. Human task crafting. It might be clear why the introduction of AI alters the tasks that have become hybrid; however, the disruption of tasks that are not related to AI is less evident. In fact, this disruption spills over from change in automated tasks, which are rarely isolated and which impact non-automated ones that could for instance be added to support AI tasks or modified to integrate them. Indeed, automation of any task can ripple out to other human tasks, especially those embedded in a same process [45]. Within the task crafting ripple, we therefore include a sub-ripple effect from hybrid to human tasks and propose four forms of human task crafting.

Add new tasks: As part of task crafting, workers might take on tasks not originally in their job's task set with the purpose of either promotion of benefits from AI or prevention of harm of AI [25]. In both cases, new task addition is a manifestation of primary control effecting external changes. When coupled with trust, automation becomes an opportunity for engaging in more meaningful tasks [17] and for seeking out new projects [25]. An example is a financial analyst relieved from the burden of analytical tasks who engages in customer financial coaching. In case of mistrust, workers are likely to engage in preventive task crafting thus adding tasks that inhibit the harm caused by AI or fix its mistakes.

Modify tasks: One of automation's most important consequences is the emergence of task reengineering [19]. Through modifying their tasks, some workers seek to increase the challenge on the job and therefore resort to job crafting [48]. One way to achieve this is through adding complexity to tasks or increasing their scope [25]. For example, nurses in a robotic surgery context can modify the prepping task from simple scrub work to setting up for robot operation. This is in line with an obtaining control process, but the same 
can be said of other control processes such as savoring for instance. In this regard, the nurses could choose to reduce the scope of their prepping task to just setting up the instrument table [49].

Remove tasks: As AI replaces some of the less cognitive or more data intensive tasks, the worker removes these tasks from their job to either go on to add new ones (see "add new tasks") or not. Depending on the control process adopted, the removal can serve to reduce workload and stress (savoring), to open the door for new opportunities of gain (obtaining) or containment (avoiding), or to withdraw (bearing).

(De)emphasize tasks: As a result of AI task performance, certain existing human tasks take on more importance than others for the worker. One reason could be that they are not replaceable by machines. An AI-assisted credit officer at the bank for instance might dedicate less time to screening loan requests and assessing risk ratios and more time on meeting with clients and researching new products. Again, the choice of task to (de)emphasize is guided by and serves the adopted control process.

\subsection{Ripple 2: Skill crafting}

A worker's pre-AI skill set might influence their choices in initial task crafting; however, as tasks are crafted, the needed skills evolve and are likely to trigger skill building behavior to accommodate the new task distribution. The skill variety and use contribute to the sense of control and eventually to a well-designed job [17]. Nevertheless, not all employees engage in self-initiated skill building. We hereby propose three skill crafting behaviors.

Upskill: The need for competence drives new skill crafting, whether it is promotion or prevention oriented [25]. As AI renders certain skills at work obsolete, task augmentation and task delegation increase the need for skills that are either more complex such as analytical skills or distinctly human such as soft and communication skills $[46,50]$. In that regard, workers following an obtaining control process are more likely to seize opportunities to update their abilities with an improved skillset. This proactive behavior is particularly valuable in supplementing efforts by organizations as skill training is becoming challenging in scale and in length. In such a demanding context, a better adaptation is achieved when one adopts a continuous learning ethos at work and anticipates changes in skill needs, thus enhancing one's sense of control [50].

Emphasize existing skills: Depending on the task crafting activities a worker engages in, the need is sometimes less to build new skills and more to increase mastery in existing ones. A radiologist for instance who distrusts diagnostic decisions of AI and who might no longer have the alternative of the older Computer Aided Diagnosis (CAD) system might need to exercise their image analysis skills to a higher level of mastery to nurture their sense of control.

None - deskill: Especially in situations where the human is out of the loop (typically delegation and withdrawal as described in task crafting), individuals who stop using certain skills needed for automated tasks risk the erosion of these skills. For example, accidents have been caused by inappropriate reactions of pilots when the autopilot did not operate as expected [17]. Skill loss or deskilling is an unintended risk of control processes involving secondary control, namely savoring and bearing.

\subsection{Ripple 3: Relational crafting}

An altered set of tasks and their associated skills result in changes to the nature and extent of interactions with people, in other terms in relational crafting [33]. Relational crafting is a particularly important and often neglected part of job crafting. Effectively, choices one makes in strengthening certain relationships and weakening others influence the kind of contagion one is exposed to. Research suggests a crossover of job crafting through learning from observation, imitation, vicarious experience, and persuasion [31]. Individuals adapt their behavior and beliefs to their social environment, as the social context makes some information more or less salient and influences expectations and even needs of which the need for control [51]. In particular, trust development is influenced by others [42] which then alters AI delegation decisions [43] and could change the course of job crafting. We propose two types of relational crafting that contribute to control.

Empowerment network: Individuals can associate through relational crafting with powerful others with the purpose of either containing AI (avoiding control) or benefiting from it to expand (obtaining control) [38]. In the context of an AI disruption, these are others who are in control, and joining in their network leads to sharing in their control through identification with the group and/or deindividuation (desire to fit in) [39]. While empowerment networks can spill over control for both negative and positive outcomes, contagion is stronger when expecting positive outcomes from imitation as in the case of obtaining and savoring control processes. Indeed, research also suggests that relationships for seeking resources or challenges at work contribute to individual adaptivity and success in change implementation [31].

Support network: Another direction in relational crafting is in the form of strengthening networks for 
support purposes. This is most aligned with a bearing control process where individuals seek relationships that increase their ability to overcome problems [38]. A worker for instance might strengthen relationships with likeminded colleagues who all distrust AI and avoid using it and where this worker seeks as well as provides support as a way of feeling in control.

\subsection{Ripple 4: Cognitive crafting}

The last ripple that results from the combination of task, skill, and relational crafting is, unlike its predecessors, of a cognitive rather than behavioral nature. As in any ripple effect, all ripples start from a common center where the disruption occurred, and this is also true for cognitive crafting. Perception of the job and the role it is associated with start as early as delegation decisions to AI are made leading to transfer of rights and responsibilities [21]. This transfer keeps evolving throughout all ripples of job crafting, not only between the AI agent and the human agent, but also among human agents.

A powerful aspect of cognitive crafting is its domain independence. On one hand, it changes the boundaries of how one sees their job, such as focusing on what they consider important or integrating it with a broader context [22]. On the other, workers can alter how they see tasks, how they judge their skills and their skill match with their job, how they think about colleagues, as well as how they perceive their whole job [25]. In broad categories, it can result in a perception that enhances the job (job expansion), narrows its boundaries (job reduction), or strives to keep it the same (job preservation).

Cognitive crafting acts as a bridge between desired and actual work outcomes. Any of the three perceptions, if aligned with desired work outcomes, leads to a perception of human-in-control, thus helping the individual deal with the adversity of a distrusted AI or increase motivation to work with a trusted one [52]. If the sense of control is not reached (e.g. cognitive crafting resulting in job reduction while the individual desires to keep the old job elements and boundaries that were disrupted by $\mathrm{AI}$ ), the pressure increases. and job crafting is likely to increase along with it [53].

\subsection{Job crafting strategies for human-in- control}

Research suggests that employees are desirous of and persistent in trying to restore their control balance [54]. The need for a sense of control is so strong in humans that individuals are likely to keep shifting from one method to the other until they reach it.
Multiple iterations of the job crafting process are thus not just possible but rather highly likely. Typically, it is expected that the first iteration(s) be one(s) involving primarily attempts at primary control. When primary control proves insufficient because of either individual or contextual factors, workers are likely to resort in later iterations to secondary control [39].

In addition to expected changes between primary and secondary control, trust is also subject to change. As individual workers engage in different job crafting strategies, they have different experiences with the AI system. An initial trust might be broken by repetitive errors in the AI outcome and the worker switches from an obtaining mode to an avoiding one. Conversely, trust in AI might improve over time. Someone who starts job crafting with a bearing control process might as a result of relational crafting be influenced by others' positive AI experiences and change their AI trust level. Change in trust leads to change in delegation [43] and that individual loops back to task crafting to delegate more to AI thereby switching to a savoring control process. This and other iterations can happen at any point in the job crafting process. Indeed, perception of control can change over time, and with it the crafting activities implemented. It is not necessarily through holding on to one process, but through reaching an optimal balance among these control processes that one achieves true adaptability and a true sense of control [39].

For the sake of clarity, we hereby explain a simple job crafting strategy based on a constant control process with a linear trajectory. We use for this purpose a hypothetical example of the work of a radiologist who follows an obtaining control process. When presented with AI-generated mammography reports, she trusts their accuracy. The system she is using is an explainable-AI and the report includes some limited rationale of the recommendation logic. Still, given the importance of the decision, she goes through a quick check herself. Augmented by the AI, she now has significantly less incidence of diagnosis error (augmentation). Realizing that image analysis is largely taken care of by the AI, she dedicates more time to patients (emphasize task) and shifts her attention to interventional radiology for breast cancer, which the AI cannot perform (add new task). For this, she partners with a more experienced interventional radiologist assisting her in operations and learning from her (upskill). She builds a stronger network in this new area she is entering (empowerment network) and where she has more direct contact with patients. As a result, she views her job as one of a contributor in the treatment of and care for cancer patients rather than an expert provider of medical imaging analysis and diagnosis (job expansion). Through her strategy 
that we named Expanding with AI, she has crafted her job to serve her desired delivery of work outcomes and better adapt in an emancipatory way to the presence of AI in her work, thus reaching a sense of human-incontrol. We expect more sophisticated non-linear job crafting strategies to emerge empirically and we leave this for future research guided by the present theoretical model.

\section{Implications and future directions}

Our work answers multiple calls for research. Examples are Von Krogh's [55] call for research that considers accountability in delegating decisionmaking to AI, Rai and colleagues' [56] emphasis on the importance of studying task augmentation in human-AI hybrid contexts, calls for research on AI enabled work transformation and its associated human-AI configurations [20].

In answering these calls, we make several important contributions to research and practice. First, the fundamental contribution is in providing a muchneeded theory to guide research work on the influence of AI on jobs and the changing nature of work. In particular, we look at workers who engage in proactive adaptive behavior by altering boundaries of their job. We therefore contribute to adaptation literature through shedding light on a wholistic adaptation of work rather than on adapting the technology or adapting the use of technology. Our theory helps researchers better understand how AI integrates with the job as a whole and provides a theoretical tool for empirical studies in this direction.

Second, we contribute back to job crafting literature by extending job crafting theory. Job crafting is an adaptive process [33] where employees adapt the meaning of their work [22] or their work resources and demands [48]. The theory is agnostic as to the event that triggers crafting activities. Most models assume that job crafting depends on the volitional motivation of employees. To our knowledge, this is the first time that job crafting theory has been applied to understanding job redesign triggered by a disruption, more specifically a technological one. By introducing a technology component to job crafting, we develop a model that better explains the particularity of adaptation to AI through job redesign.

We also augment job crafting theory with a richer conceptualization of control. Particularly, we introduce the new concept of human-in-control and emphasize its importance as a goal of job crafting and an essential element in AI job transformation. Our model does not attribute control solely to situations of technology adoption. We avoid technology bias and acknowledge that a sense of control can be reached by adopters as well as individuals resisting or avoiding AI.

Our contribution to practice is no less essential as change management practices currently rely on traditional IT and need to be adapted to the new AI reality. Namely, in addition to technological considerations, managers need to place stronger emphasis on human participants. They ought to consider their needs and interests to better influence their control process development and hence their job crafting and work outcomes. Organizations can provide these managers with targeted training on how to empower employees for effective job crafting. They can also facilitate upskilling by providing adaptive or customizable training to workers and encourage favorable forms of crafting by nurturing their autonomy and building their trust in AI. Further, by providing insight into employee-initiated bottom-up changes to jobs, we help management better design their top-down HR strategies since the first informs and guides the second. Last, we contribute to practice by shedding light on the important issue of accountability and its impact on employees' proactive behavior at work. Both managers and regulators need to clarify the boundaries of accountability between humans and machines in an AI context.

Our work provides a novel and important perspective on job transformation with AI. As such, it opens numerous avenues for research in this nascent stream in the IS field. First, an important research avenue is conducting empirical studies that not only test the theory, but also adapt it to different occupations and organizational situations. Different crafting patterns are likely to emerge for various professions, positions, industries, and types of organizations to name a few. These crafting variations can also manifest in a different order of ripples. For instance, instead of starting with task crafting, some patterns might be initiated by cognitive crafting as a pre-behavioral response followed by the other forms of crafting. An empirical context can therefore bring pertinent variations to the proposed model.

Second, as we introduce the new concept of human-in-control, research that refines it and elaborates it can advance the discussion on the role of control in the human-AI relationship. In particular, an avenue that we believe to be particularly promising is human-AI collaboration. With many jobs currently augmented by AI rather than replaced by it, studying collaboration between humans and AI in a hybrid environment becomes key to understanding many organizational AI phenomena. How control influences this collaboration and whether in highly autonomous contexts secondary control can still be viable are just a few of the questions that can be investigated. Further, 
our study assumes that crafting behavior only happens on the human side. However, learning in many AI contexts is bi-directional, and research that studies the knowledge exchange between humans and AI during the crafting process and the resulting learning and crafting activity in both AI and humans provides much value for a better understanding of this hybrid form of collaboration. This brings to the fore the human-incontrol concept we introduced as it is essential in such collaboration to keep the human as the central agent.

While acknowledging the social aspect of job crafting especially in relational crafting activities, our theory is conceptualized at the individual level. We believe there is much value in exploring how job crafting in an AI context is deployed at the group and organizational levels as well as cross-level. Questions related to the network influence on job crafting strategies or to the interplay of crafting between different occupations collaborating on certain tasks are examples of such research opportunities.

These are but a few of the future research possibilities that can be facilitated by our theory, as it presents a first conceptualization of an informal job redesign by the workers themselves in response to AI disruption. We hope our paper will stimulate research on such an important phenomenon and encourage empirical studies in this novel research stream.

\section{References}

[1] Davis, F.D., "Perceived Usefulness, Perceived Ease of Use, and User Acceptance of Information Technology", MIS Quarterly, 1989, pp. 319-340.

[2] Venkatesh, V., M. Morris, G. Davis, and F. Davis, "User Acceptance of Information Technology: Toward a Unified View", MIS Quarterly 27(3), 2003, pp. 425-478.

[3] Iacovou, C.L., I. Benbasat, and A.S. Dexter, "Electronic Data Interchange and Small Organizations: Adoption and Impact of Technology", MIS Quarterly 19(4), 1995, pp. 465-485.

[4] Lapointe, L., and S. Rivard, "A Multilevel Model of Resistance to Information Technology Implementation", MIS Quarterly 29(3), 2005, pp. 461.

[5] Bala, H., and V. Venkatesh, "Adaptation to Information Technology: A Holistic Nomological Network from Implementation to Job Outcomes", Management Science 62(1), 2016, pp. 156-179.

[6] Majchrzak, A., R.E. Rice, A. Malhotra, N. King, and S. $\mathrm{Ba}$, "Technology Adaptation: The Case of a ComputerSupported Inter-Organizational Virtual Team", MIS Quarterly 24(4), 2000, pp. 569-600.

[7] Beaudry, A., and A. Pinsonneault, "Understanding User Responses to Information Technology: A Coping Model of User Adaptation", MIS Quarterly 29(3), 2005, pp. 493-524.

[8] Beaudry, A., and A. Pinsonneault, "The Other Side of Acceptance: Studying the Direct and Indirect Effects of
Emotions on Information Technology Use", MIS Quarterly 34(4), 2010, pp. 689-710.

[9] Levina, N., and E. Vaast, "The Emergence of Boundary Spanning Competence in Practice. Implications for Implementation and Use of Information Systems", MIS Quarterly 29(2), 2005, pp. 335-363.

[10] Polites, G.L., and E. Karahanna, "The Embeddedness Of Information Systems Habits In Organizational And Individual Level Routines: Development And Disruption”, MIS Quarterly 37(1), 2013, pp. 221- +.

[11] Leonardi, P., "When Flexible Routines Meet Flexible Technologies: Affordance, Constraint, and the Imbrication of Human and Material Agencies", MIS Quarterly 35(1), 2011, pp. 147.

[12] Cousins, K.C., and D. Robey, "The Social Shaping of Electronic Metals Exchanges: An Institutional Theory Perspective", Information Technology \& People, 2005.

[13] Tyre, M.J., and W.J. Orlikowski, "Windows of Opportunity: Temporal Patterns of Technological Adaptation in Organizations", Organization Science 5(1), 1994, pp. 98-118.

[14] Boudreau, M.-C., and D. Robey, "Enacting Integrated Information Technology: A Human Agency Perspective", Organization Science 16(1), 2005, pp. 3-18.

[15] Leonardi, P.M., "Activating the Informational Capabilities of Information Technology for Organizational Change", Organization Science 18(5), 2007, pp. 813-831.

[16] Rahrovani, Y., and A. Pinsonneault, "Innovative IT Use and Innovating with IT: A Study of the Motivational Antecedents of Two Different Types of Innovative Behaviors", Journal of the Association for Information Systems 21(4), 2020, pp. 936-970.

[17] Parker, S.K., and G. Grote, "Automation, Algorithms, and Beyond: Why Work Design Matters More Than Ever in a Digital World", Applied Psychology-An International Review, 2020.

[18] LeCun, Y., Y. Bengio, and G. Hinton, "Deep Learning”, Nature 521(7553), 2015, pp. 436-444.

[19] Acemoglu, D., and P. Restrepo, "Low-Skill and HighSkill Automation", Journal of Human Capital 12(2), 2018, pp. 204-232.

[20] Rahwan, I., M. Cebrian, N. Obradovich, et al., "Machine Behaviour", Nature 568(7753), 2019, pp. 477.

[21] Baird, A., and L.M. Maruping, "The Next Generation of Research on IS Use: A Theoretical Framework of Delegation to And from Agentic IS Artifacts", MIS Quarterly 45(1), 2021, pp. 315-341.

[22] Wrzesniewski, A., and J.E. Dutton, "Crafting a Job: Revisioning Employees as Active Crafters of Their Work", Academy of Management Review 26(2), 2001, pp. 179-201.

[23] Tims, M., A.B. Bakker, and D. Derks, "Development and Validation of the Job Crafting Scale", Journal of Vocational Behavior 80(1), 2012, pp. 173-186.

[24] Demerouti, E., "Design Your Own Job Through Job Crafting", European Psychologist 19(4), 2014, pp. 237247.

[25] Bindl, U.K., K.L. Unsworth, C.B. Gibson, and C.B. Stride, "Job Crafting Revisited: Implications of an Extended fFamework for Active Changes at Work.", Journal of Applied Psychology 104(5), 2018, pp. 605-628. 
[26] Bruning, P.F., and M.A. Campion, "A Role-resource Approach-avoidance Model of Job Crafting: A Multimethod Integration and Extension of Job Crafting Theory", Academy of Management Journal 61(2), 2018, pp. 499-522.

[27] Wrzesniewski, J. Berg, A. Grant, J. Kurkoski, and B. Welle, "Job Crafting in Motion: Achieving Sustainable Gains in Happiness and Performance", Annual Meeting of the Academy of Management, Boston, MA, (2012).

[28] Brynjolfsson, E., and A. McAfee, "The Business of Artificial Intelligence", Harvard Business Review, 2017. https://hbr.org/2017/07/the-business-of-artificialintelligence

[29] Burrell, J., "How the Machine 'Thinks': Understanding Opacity in Machine Learning Algorithms", Big Data \& Society 3(1), 2016, pp. 2053951715622512.

[30] Grant, A.M., and S.K. Parker, "7 Redesigning Work Design Theories: The Rise of Relational and Proactive Perspectives", The Academy of Management Annals 3(1), 2009, pp. 317-375.

[31] Peeters, M.C.W., R. Arts, and E. Demerouti, "The Crossover of Job Crafting Between Coworkers and Its Relationship with Adaptivity", European Journal of Work and Organizational Psychology 25(6), 2016, pp. 819-832.

[32] Lazazzara, A., M. Tims, and D. De Gennaro, "The Process of Reinventing a Job: A Meta-Synthesis of Qualitative Job Crafting Research", Journal of Vocational Behavior 116, 2020, pp. 103267.

[33] Berg, J.M., A. Wrzesniewski, and J.E. Dutton, "Perceiving and Responding to Challenges in Job Crafting at Different Ranks: When Proactivity Requires Adaptivity", Journal of Organizational Behavior 31(23), 2010, pp. 158-186.

[34] Lerner, J.S., and P.E. Tetlock, "Accounting for the Effects of Accountability", Psychological Bulletin 125(2), 1999, pp. 255-275.

[35] Schönberger, D., "Artificial Intelligence in Healthcare: A Critical Analysis of the Legal and Ethical Implications", International Journal of Law and Information Technology 27(2), 2019, pp. 171-203.

[36] Holzinger, A., "Interactive Machine Learning for Health Informatics: When Do We Need the Human-inthe-Loop?", Brain Informatics; Heidelberg 3(2), 2016, pp. 119-131.

[37] Bengio, Y., T. Deleu, N. Rahaman, et al., “A MetaTransfer Objective for Learning to Disentangle Causal Mechanisms", arXiv:1901.10912 [cs, stat], 2019.

[38] Bryant, F.B., "A Four-Factor Model of Perceived Control: Avoiding, Coping, Obtaining, and Savoring", Journal of Personality 57(4), 1989, pp. 773-797.

[39] Rothbaum, F., J. Weisz, and S. Snyder, "Changing the World and Changing the Self - A 2-Process Model of Perceived Control", Journal of Personality And Social Psychology 42(1), 1982, pp. 5-37.

[40] Dimoka, A., "What Does the Brain Tell Us About Trust and Distrust? Evidence from a Functional Neuroimaging Study", MIS Quarterly 34(2), 2010, pp. 373-396.

[41] Abbass, H.A., "Social Integration of Artificial Intelligence: Functions, Automation Allocation Logic and Human-Autonomy Trust", Cognitive Computation 11(2), 2019, pp. 159-171.

[42] Glikson, E., and A.W. Woolley, "Human Trust in Artificial Intelligence: Review of Empirical Research", Academy of Management Annals(ja), 2020.

[43] Castelfranchi, C., and R. Falcone, "Trust and Control: A Dialectic Link", Applied Artificial Intelligence 14(8), 2000, pp. 799-823.

[44] Dellermann, D., P. Ebel, M. Soellner, and J.M. Leimeister, "Hybrid Intelligence", Business \& Information Systems Engineering 61(5), 2019, pp. 637-643.

[45] Raisch, S., and S. Krakowski, "Artificial Intelligence and Management: The Automation-Augmentation Paradox", Academy of Management Review 46(1), 2021, pp. 192-210.

[46] McGuinness, S., K. Pouliakas, and P. Redmond, "SkillsDisplacing Technological Change and Its Impact on Jobs: Challenging Technological Alarmism?", Economics of Innovation and New Technology, 2021, pp. 1-23.

[47] Shrestha, Y.R., S.M. Ben-Menahem, and G. von Krogh, "Organizational Decision-Making Structures in the Age of Artificial Intelligence", California Management Review 61(4, SI), 2019, pp. 66-83.

[48] Tims, M., and A.B. Bakker, "Job Crafting: Towards a New Model of Individual Job Redesign", SA Journal of Industrial Psychology 36(2), 2010, pp. 1-9.

[49] Sergeeva, A., M. Huysman, and S. Faraj, "Transforming Work Practices of Operating Room Teams: The Case of the Da Vinci Robot", ICIS (2015).

[50] Willcocks, L., "Robo-Apocalypse Cancelled? Reframing the Automation and Future of Work Debate", Journal of Information Technology 35(4), 2020, pp. 286-302.

[51] Salancik, G., and J. Pfeffer, "Social InformationProcessing Approach to Job Attitudes and Task Design", Administrative Science Quarterly 23(2), 1978, pp. 224253.

[52] Buonocore, F., D. de Gennaro, M. Russo, and D. Salvatore, "Cognitive Job Crafting: A Possible Response to Increasing Job Insecurity and Declining Professional Prestige", Human Resource Management Journal 30(2), 2020, pp. 244-259.

[53] Wang, H.-J., E. Demerouti, P. Le Blanc, and C.-Q. Lu, "Crafting a Job in "Tough Times': When Being Proactive is Positively Related to Work Attachment", Journal of Occupational and Organizational Psychology 91(3), 2018, pp. 569-590.

[54] Greenberger, D., and S. Strasser, "Development and Application of a Model of Personal Control in Organizations", Academy of Management Review 11(1), 1986, pp. 164-177.

[55] von Krogh, G., "Artificial Intelligence in Organizations: New Opportunities for Phenomenon-Based Theorizing", Academy of Management Discoveries 4(4), 2018, pp. 404409.

[56] Rai, A., P. Constantinides, and S. Sarker, "NextGeneration Digital Platforms: Toward Human-AI Hybrids", MIS Quarterly 43(1), 2019, pp. iii-ix. 